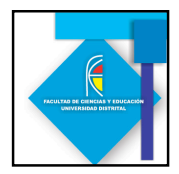

\title{
EL PAPEL DE LA OBSERVACIÓN EN LA FORMALIZACIÓN DE CONCEPTOS A PARTIR DE LA CONTRIBUCIÓN DE LA ASTRONOMÍA. CASO PARTICULAR: NUESTRA ESTRELLA EL SOL.
}

\section{THE OBSERVATION ROLE IN FORMALIZATION OF CONCEPTS FROM THE ASTRONOMY CONTRIBUTION. PARTICULAR CASE: OUR STAR, THE SUN.}

\author{
Marlón Arias ${ }^{1}$ \\ Andrea Huerfano ${ }^{2}$
}

\begin{abstract}
Resumen
Este trabajo presenta una estrategia pedagógica para el desarrollo de la observación como método de investigación en estudiantes de básica primaria, con el fin de promover la comprensión de fenómenos físicos, a partir de la exploración de las características básicas del Sol. Su objetivo principal es potencializar los dispositivos básicos de aprendizaje en estudiantes de primaria, a partir del desarrollo de actividades que promuevan un adecuado desenvolvimiento de los niños dentro y fuera del entorno escolar. Se busca trabajar a partir del reconocimiento y la constante observación de ideas en torno al sol que han construido los estudiantes e introducir relaciones que posibiliten un dialogo desequilibrante entre maestro-estudiante y estudiante-estudiante sobre fenómenos relacionados al sol como lo son: las sombras, porque hay día y noche, la apariencia y composición del sol, entre otras. Al mismo tiempo, posibilitar la incorporación de material de trabajo de las ciencias físicas básicas, junto con actividades artísticas que apoyen el desarrollo del razonamiento espacial y lógico, en búsqueda de estimular a los estudiantes hacia el conocimiento de elementos en astronomía y que ellos logren dar razón de fenómenos que suceden a su alrededor y encontrar respuestas de sucesos que se den en su quehacer cotidiano.
\end{abstract}

Palabras clave: Sol, observación, formalización.

\footnotetext{
Abstract

This paper presents a pedagogical strategy for the development of observation as a research method in elementary school students in order to promote understanding of

${ }^{\text {I}}$ Estudiante de Licenciatura en Psicología y Pedagogía Universidad Pedagógica Nacional. apache141@hotmail.com

Estudiante Licenciatura en Física Universidad Pedagógica Nacional. anmarphy@gmail.com
} 
$5^{\circ}$ CONGRESO NACIONAL DE ENSEÑANZA DE LA FÍSICA.

Universidad Pedagógica Nacional (U. P. N)

Universidad Distrital Francisco José de Caldas (U. D. F. J. C)

Bogotá, Colombia. 16 al 20 de mayo 2011

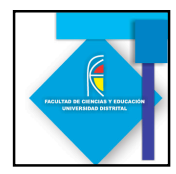

physical phenomena, based on the exploration of the basic characteristics of the sun, its main objective potentiate the basic devices of learning in basic elementary students from the development of activities to promote in a proper development of children inside and outside of the school environment. It looks for to build in recognition and constant observation of ideas around the sun that students have built in students to relation to and introduce to enable a dialogue between teacher-student destabilizing and student-student on sun-related phenomena such as: the shadows, the existence of day and night, the appearance and composition of the sun, among others. At the same time, facilitate the incorporation of materials used for basic physics science, along with art activities that support the development of spatial and logic reasoning, seeking to encourage students to knowledge of astronomy and elements to achieve them to answer phenomena's that happens around and find answers to event that occur in their diary activities.

Keywords: Sun, Observation, Formalization.

\section{Introducción}

Los estándares básicos en competencias en ciencias naturales y sociales promueven la aproximación de la ciencia a todos los grados de escolaridad a partir de la formulación de preguntas, conjeturas e hipótesis que surgen como resultado de la observación del entorno [1]. Es por este motivo, que proponemos una serie de actividades involucradas con el sol y dirigidas a niños de básica primaria, donde se privilegie la observación en la formalización de conceptos.

Además, se busca resaltar la capacidad de asombro de los niños, la cual se manifiesta en la curiosidad por conocer y el tratar de comprender el mundo que les rodea. Por este motivo, la importancia de estimular la formación científica desde una edad muy temprana para potencializar el desarrollo de las habilidades del pensamiento involucradas en el acto de observación como lo son: percibir, identificar y discriminar; las cuales conducen el proceso cognitivo hasta la formalización de conceptos como resultado de transformaciones en las representaciones construidas.

De esta manera, nuestra intención es aprovechar la curiosidad reconocida en los niños e integrar los objetivos planteados en los estándares de educación, para diseñar una propuesta pedagógica, basada en un conjunto de actividades secuenciales de diferentes grados de abstracción, cuyo propósito sea ampliar las comprensiones de los niños respecto a la relación Tierra-Sol.

\section{Astronomía: Una experiencia significativa}

A lo largo de la historia el hombre se ha interesado por comprender las dinámicas de su entorno, cautivándose por lo desconocido e inalcanzable. Muestra de esto, es el deseo que surge por conocer los astros que aparecen en el cielo, un claro ejemplo lo constituye el interés que despertó en un amplio número de culturas la Luna y el Sol, los cuales fueron considerados deidades y en su nombre se realizaron ofrendas, ceremonias y templos.

No obstante, hoy en día este interés sigue presente y no es de extrañar que se indague la manera de llevar estos contenidos al aula. Es por este motivo, que se ha venido diseñando 
$5^{\circ}$ CONGRESO NACIONAL DE ENSEÑANZA DE LA FÍSICA.

Universidad Pedagógica Nacional (U. P. N)

Universidad Distrital Francisco José de Caldas (U. D. F. J. C)

Bogotá, Colombia. 16 al 20 de mayo 2011

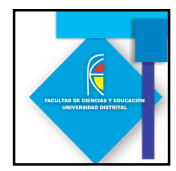

material didáctico no solo en universidades que ofrecen licenciaturas, sino también en institutos y laboratorios de física, como por ejemplo el CSE -Center for Science Education- del Laboratorio de Ciencias Espaciales de la Universidad de Berkeley y de California, quienes financiados por la NASA diseñaron un conjunto de 7 guías dirigidas a maestros sobre magnetismo aplicado a fenómenos astronómicos [2]. La NASA de manera independiente ha diseñado unidades didácticas con el objetivo de facilitar la introducción de temas de astronomía en la escuela, como es el caso de la cartilla "Our Eye on the Sun", la cual intenta presentar de manera sencilla una sólida fundamentación de la ciencia, que integra las propuestas señaladas a abarcar en enseñanza de la misma según los Elementary National Standars con respecto a la relación Tierra-Sol.

Las iniciativas mencionadas son el resultado de indagaciones orientadas al proceso de alfabetización científica en beneficio de "la comprensión profunda de las características y leyes básicas del mundo que nos rodea" [3]. Por lo tanto, consideramos que las actividades involucradas con temáticas de astronomía permiten desarrollar comprensiones significativas del mundo en el niño, debido a que dan explicación a fenómenos que suceden a su alrededor y son sencillos de percibir.

\section{El papel de la observación en la formalización de conceptos.}

En esta propuesta prevalece la observación como una operación mental integradora que permite la formalización de conceptos, al involucrar de manera implícita habilidades como: la percepción, la identificación, la discriminación y la caracterización. Habilidades que son de gran importancia en la construcción de ideas o representaciones, que dan paso al desarrollo de estructuras cognitivas y a la formalización de conceptos.

En este sentido, nos parece pertinente plantear una propuesta orientada desde el constructivismo, debido a que permite valorar "el intento por comprender como los alumnos han construido un sistema categorial para aprender los nuevos conocimientos" [4]. Además, porque desde este enfoque psicológico podemos encontrar diversos argumentos para generar espacios que desarrollen un método de enseñanza de los conceptos a través de la percepción e interacción con los objetos, que en el caso de nuestra propuesta, seria la interacción del estudiante con el medio.

Para esta propuesta, soportamos el porqué trabajar en niños de cuarto grado de una institución educativa, desde la teoría del desarrollo de Jean Piaget, quien dice que los niños que se encuentran entre los 7 y 11 años (Referencia de edad que en la actualidad, nos ubica en su mayoría a niños que cursan en el sistema colombiano de educación, el grado cuarto y quinto de básica primaria), se desenvuelven en la etapa de las operaciones concretas. Lo cual implica que están en una época del desarrollo cognoscitivo caracterizado por el desarrollo de esquemas mentales para "las operaciones lógicas de seriación y calificación, y se perfeccionan los conceptos de causalidad, espacio, tiempo y velocidad" [5], necesarios para la formalización de conceptos e inicio de un proceso de alfabetización científica.

En esta etapa de desarrollo propuesta por Piaget, el niño ya no está supeditado a la percepción, toma en cuenta las transformaciones, desarrolla operaciones lógicas (la reversibilidad, la clasificación, etc.) que le son útiles en la solución de problemas, 
$5^{\circ}$ CONGRESO NACIONAL DE ENSEÑANZA DE LA FÍSICA.

Universidad Pedagógica Nacional (U. P. N)

Universidad Distrital Francisco José de Caldas (U. D. F. J. C)

Bogotá, Colombia. 16 al 20 de mayo 2011



comprensión de objetos y sucesos concretos (reales, observables) [5], por esta razón, ya se puede programar la observación no solo como una operación mental integradora como decimos al inicio de este apartado, sino como un método de investigación que integre los conocimientos previos y genere desafíos cognitivos a los cuales hay que buscar respuesta.

\section{Desarrollo de la estrategia}

Resaltando el papel de la observación como potencializadora de habilidades de pensamiento que conduzcan a la formalización de conceptos, en un espacio escolar con participación activa de los estudiantes en torno a un eje temático específico. Se planeta en inicio desarrollar la propuesta en tres sesiones, cada una de 60 minutos aproximadamente, dividida en momentos puntuales que van a favorecer la comprensión, el análisis y la asimilación de conceptos.

A continuación se esbozan las tres sesiones:

Sesión 1: "Explorando la relación tierra - sol"

Inicialmente se reconocen las ideas que tienen los estudiantes respecto al sol, por medio de preguntas como: ¿Dónde está el sol? ¿Por qué crees que brilla el sol? ¿De que esta hecho? ¿Cómo es el sol? Se propone para esta última pregunta que los niños dibujen el sol, para identificar la representación visual que han construido desde su experiencia. En un segundo momento, se presentan varias imágenes de estrellas para mostrar que el sol hace parte de una clase especifica de cuerpos celestes, de esta manera se amplía en el niño sus nociones respecto al espacio y se hace una breve reseña de la manera como se obtienen estas fotografías. Además se motiva a que los niños discriminen y comparen las imágenes para emitir juicios de valor que ayuden a esclarecer una posible definición y caracterización del sol.

Sesión 2: "Construyo desde mi experiencia correteando con las estrellas"

Para establecer relaciones entre tamaño y distancia de la Tierra y el Sol. Se propone el uso de analogías entre objetos que permitan bajar la escala del sol y hacerla cercana para el niño. Procediendo en la introducción de fenómenos para formalizar conceptos, se establecen relaciones entre el día y la noche respecto al papel del sol, y se propone la lectura del cuento "Los limpiadores de estrellas" escrito por Julio Cortázar con el objetivo de puntualizar relaciones respecto al papel de la luminosidad de las estrellas en el día y la noche.

\section{Sesión 3: "Jugando y aprendiendo con las sombras"}

Para terminar la secuencia de actividades se propone inicialmente una presentación del movimiento de la tierra alrededor del sol, para proceder con la observación directa de las sombras a fin de responder a preguntas como: ¿Cómo se producen las sombras? ¿Las sombras cambian de forma? ¿Cómo se relaciona el sol con las sombras? Proponiéndose el dibujo de las sombras de animales, objetos y humanos cuidadosamente a lo largo del día, para comparar su forma. 
$5^{\circ}$ CONGRESO NACIONAL DE ENSEÑANZA DE LA FÍSICA.

Universidad Pedagógica Nacional (U. P. N)

Universidad Distrital Francisco José de Caldas (U. D. F. J. C)

Bogotá, Colombia. 16 al 20 de mayo 2011

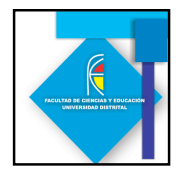

Como cierre de las sesiones se procederá a la elaboración artística del sol con materiales de fácil acceso y bajo costo, de manera que el niño materialice la nueva representación construida respecto al sol.

\section{Evaluación del aprendizaje}

Compartiendo la visión de evaluación de la pedagogía critica, entendemos la evaluación como un proceso de acompañamiento permanente que complementa la formación de los estudiantes y permite mejorar el proceso a partir de estrategias que no se quedan en calificar, sino que van encaminadas al desarrollo de la capacidad de argumentación, que en este caso, será la de fenómenos implicados en la relación tierra-sol.

Para lo anterior, debemos tener en cuenta como guía de nuestro trabajo, que "la simple inquietud compartida provoca procesos de reflexión sobre la práctica, capaces de facilitar la comprensión y de modificar el lenguaje, las relaciones y los modos de acción"[6], permitiéndonos un mejor análisis del proceso de aprendizaje de cada estudiante y practicar momentos de retroalimentación en cada sesión, lo que constituye un proceso de acompañamiento permanente y fundamental durante el proceso.

\section{Conclusiones}

En pocas palabras, la astronomía es un medio de conocimiento que estimula al estudiante a conocer su mundo y a explicar lo que pasa en el. La enseñanza de la astronomía a una menor edad, permite que al mismo tiempo que se explore el entorno, se generen representaciones mentales y se desarrollen en el estudiante sus gnosias, psicomotricidad y percepción, a través de la observación y la vivencia del saber que en inicio es concreto y requiere de una abstracción posterior.

Para terminar, el papel de la observación es fundamental para poder comprender la contribución de la astronomía en la formulación de conceptos, pues bien, "Observar es descubrir cosas, es parte de un proceso de reaccionar significativamente ante el mundo" [7].

\section{Referencias Bibliográficas}

[1] Ministerio de Educación Nacional, Estándares Básicos de Competencias en Ciencias Naturales y Ciencias Sociales. Formar en ciencias iel desafío! Lo que necesitamos saber y hacer. Disponible: http://www.mineducacion.gov.co/1621/articles-81033_archivo_pdf.pdf

[2] The Center for Science Education (CSE) at the UC Berkeley Space Science Laboratory; National Aeronautics and Space Administration (NASA). Exploring Magnetism. Lesson Series. http://cse.ssl.berkeley.edu/segwayed/lessons/exploring_magnetism/index.html 




[3] Alfabetización científica: cómo, cuándo y por qué. Por Melina

Furman. Disponible: http://planetciencia.blogspot.com/2010/11/alfabetizacioncientifica-como-cuando-y.html

[4] CAMILLONI, Alicia R.W; CELMAN, Susana; LITWIN, Edith y PALAU DE MATÉ M. del Carmen (1998). La evaluación de los aprendizajes en el debate didáctico contemporáneo. Buenos Aires: Paidós.

[5] WADSWORTH, Barry J. (1991) Teoría de Piaget del Desarrollo Cognoscitivo y Afectivo. DIANA. Pág. 104

[6] SANTOS GUERRA, Miguel Ángel (2003) Trampas en educación, el discurso sobre calidad. La Muralla S.A. Pág. 50

[7] RATHS, Louis Edward (1971) Como enseñar a pensar: teoría y aplicación. Buenos Aires. Paidos. Pág. 30 